\begin{tabular}{|c|c|}
\hline Title & Intensified and attenuated waves in a microbubble Taylor-Couette flow \\
\hline Author(s) & W atamura, T.; T asaka, Y .; Murai, Y. \\
\hline Citation & $\begin{array}{l}\text { Physics of Fluids, } 25(5), 054107 \\
\text { https://doi.org/10.1063/1.4804392 }\end{array}$ \\
\hline Issue Date & $2013-05$ \\
\hline Doc URL & http:/hdl.handle.net/2115/53120 \\
\hline Rights & $\begin{array}{l}\text { Copyright } 2013 \text { A merican Institute of Physics. This article may be downloaded for personal use only. A ny other use } \\
\text { requires prior permission of the author and the A merican Institute of Physics. The following article appeared in Phys. } \\
\text { Fluids } 25,054157 \text { (2013) and may be found at http:/pof.aip.org/resource//phfle6 } / 25 / 5 / p 054107 \text { s1 }\end{array}$ \\
\hline Type & article \\
\hline File Information & PhysFluids_25_054107.pdf \\
\hline
\end{tabular}

Instructions for use 


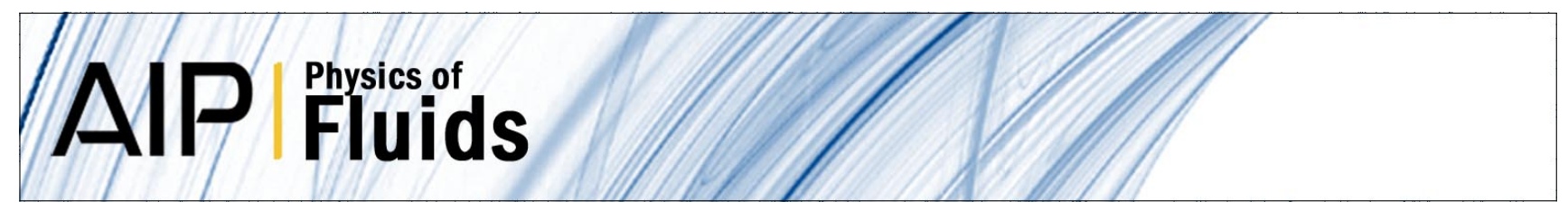

\section{Intensified and attenuated waves in a microbubble Taylor-Couette flow}

T. Watamura, Y. Tasaka, and Y. Murai

Citation: Phys. Fluids 25, 054107 (2013); doi: 10.1063/1.4804392

View online: http://dx.doi.org/10.1063/1.4804392

View Table of Contents: http://pof.aip.org/resource/1/PHFLE6/v25/i5

Published by the AIP Publishing LLC.

\section{Additional information on Phys. Fluids}

Journal Homepage: http://pof.aip.org/

Journal Information: http://pof.aip.org/about/about_the_journal

Top downloads: http://pof.aip.org/features/most_downloaded

Information for Authors: http://pof.aip.org/authors

\section{ADVERTISEMENT}

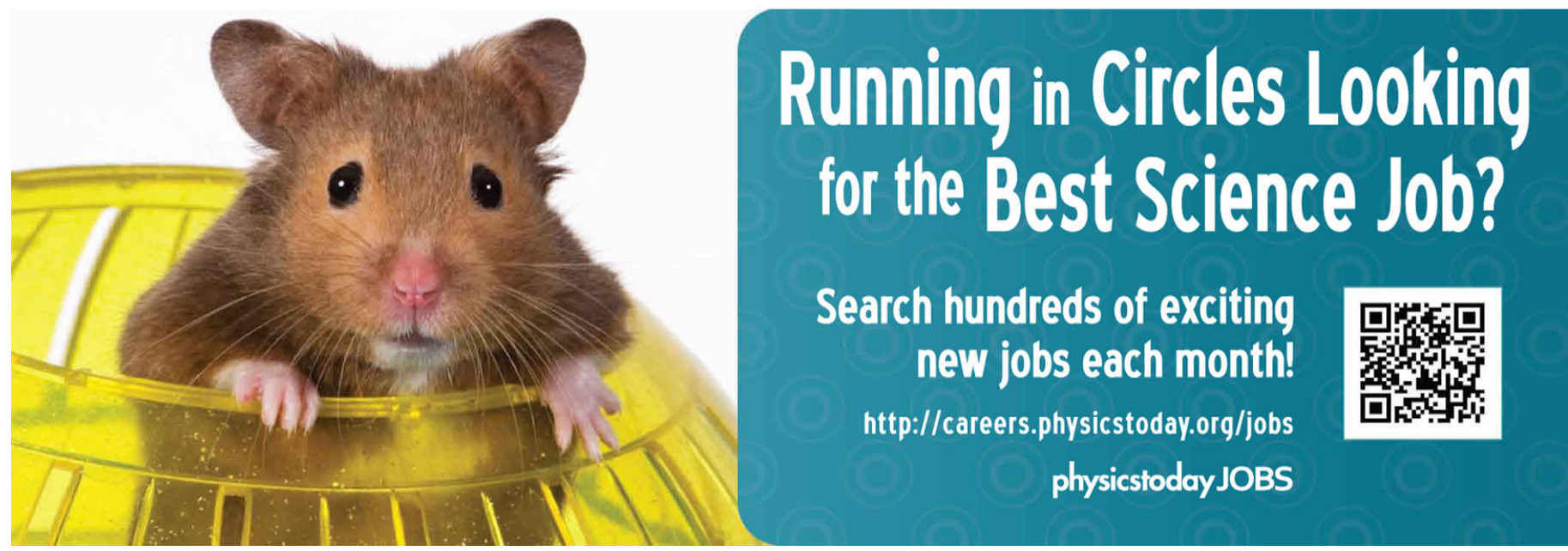




\title{
Intensified and attenuated waves in a microbubble Taylor-Couette flow
}

\author{
T. Watamura, ${ }^{a)}$ Y. Tasaka, and Y. Murai \\ Laboratory for Flow Control, Graduate School of Engineering, Hokkaido University, \\ Kita-13 Nishi-8, Sapporo 060-8628, Japan
}

(Received 27 September 2012; accepted 5 April 2013; published online 20 May 2013)

\begin{abstract}
The effect of the presence of microbubbles on a flow state is experimentally investigated in a Taylor-Couette flow with azimuthal waves, in order to examine the interaction mechanism of bubbles and flows that result in drag reduction. The average diameter of the bubbles is $60 \mu \mathrm{m}$, which is smaller than the Kolmogorov length scale, and the maximum void fraction is $1.2 \times 10^{-4}$ at the maximum case. The modifications of the fluid properties, bulk density, effective viscosity, and the extra energy input caused by the addition of microbubbles are expected to have a small effect on modifying flow states. The power of the basic wave propagating in the azimuthal direction is enhanced; its modulation, however, is decreased by adding microbubbles in the flow regime corresponding to modulated Taylor vortex flow. Moreover, the gradient of the azimuthal velocity near the walls, source of the wall shear stress, decreases by $10 \%$. The modified velocity distribution by adding microbubbles is comparable to that obtained with a 20\% lower Reynolds number. Microbubbles in the coherent structure of the wavy Taylor vortices are visualized and exhibit a preferential distribution and motion at the crests and troughs of the waviness. The roles of the inhomogeneously distributed microbubbles in wavy vortical structures are discussed in view of our findings. ( 2013 AIP Publishing LLC. [http://dx.doi.org/10.1063/1.4804392]
\end{abstract}

\section{INTRODUCTION}

Injecting bubbles as a way for skin frictional drag reduction in shear flows has been considered for engineering applications, particularly for large vessels. ${ }^{1}$ Drag reduction by the injection of $O(10 \mu \mathrm{m})$ diameter bubbles (hereafter termed as "microbubbles") into turbulent boundary layers has been a subject of intensive research since the first experimental investigation ${ }^{2}$ because of its high efficiency, i.e., drag reduction rate versus the input void fraction. The performance of the drag reduction rate has been experimentally investigated in turbulent channel flows ${ }^{3-7}$ or in turbulent boundary layers on a flat wall, ${ }^{8}$ and reaches $\sim 40 \%$ at the maximum. The remarkable feature of drag reduction by microbubbles is that $O(10 \%)$ of the drag reductions are achieved by low void fractions of $\alpha \sim O\left(10^{-4}\right)$.

As a fundamental approach for understanding the drag reduction mechanisms, modifications of the liquid medium property in bulk have been considered as the function of the void fraction so far. The modification on the bulk density of liquids is given as

$$
\rho=(1-\alpha) \rho_{0},
$$

where $\rho_{0}$ is the original density of the liquids. The air density of bubbles is much smaller than that of the liquids and is regarded as zero. The decrease in the medium's density with increasing void fraction provides the primary mechanism for drag reduction with decreasing the bulk density of the medium in turbulence. ${ }^{9}$ In contrast, the effective (bulk) viscosity of the fluid is estimated by Taylor's

\footnotetext{
a) twatamura@ ring-me.eng.hokudai.ac.jp
} 
formula, ${ }^{10,11}$

$$
\mu=(1+\alpha) \mu_{0},
$$

where $\mu_{0}$ is the viscosity of the liquids, and it yields the opposite relationship, i.e., an increase in the shear stress. An increase in the void fraction decreases the frictional drag by decreasing the Reynolds shear stress with decreasing the medium density, as described in Eq. (1), and increases it by increasing the effective viscosity at the moment, according to Eq. (2). The linear theory that resolves this contradiction states that the modification on the properties of fluids with uniformly dispersed bubbles is of the same order as the void fraction. ${ }^{12}$ Further, the energy relationship for systems containing bubbles in an isentropic process is given as

$$
\langle\bar{E}\rangle=\langle\bar{\varepsilon}\rangle-\left\langle\overline{\boldsymbol{f}_{\text {bubble }} \cdot \boldsymbol{u}}\right\rangle,
$$

where $E, \varepsilon$, and $f_{\text {bubble }}$ are the energy input rate, energy dissipation rate, and extra energy input to the system due to the momentum transfer of bubbles, respectively. The angle brackets, \langle\rangle , and overline, ${ }^{-}$, indicate spatial and time averages, respectively. Assuming that changes in the energy relationship are caused by the sum of the buoyant forces acting on each microbubble dispersed in the liquid phase,

$$
\left\langle\overline{\boldsymbol{f}_{\text {bubble }} \cdot \boldsymbol{u}}\right\rangle \approx-\alpha \mathbf{g} \cdot\left\langle\overline{\boldsymbol{u}_{\text {bubble }}}\right\rangle,
$$

and the microbubbles should modify the momentum transfer very little or not at all, ${ }^{13}$ if the mean void fraction is as small as $O(0.1 \%)$. The effects of the modification in density, effective viscosity, and extra energy input would be of the same order as the void fraction, and would be negligible in dilute two-phase flows, i.e., $\alpha \leq O\left(10^{-4}\right)$. The drag reduction rate in previous experimental investigations of dilute two-phase flows containing microbubbles, ${ }^{4,7,8}$ in contrast, takes significantly high values since nonlinear interactions occur between microbubbles and flow structures in turbulent flows, as mentioned by L'vov et al. ${ }^{12}$ Investigations of flow fields in the liquid phase indicate that the drag reduction mechanisms can now be attributed to a decrease in the coherence of near wall structure (the source of Reynolds shear stress), which arises from interactions between microbubbles and coherent flow structures. ${ }^{5-8}$ Numerical works as well as experiments exhibited the decrease in the Reynolds shear stress near the wall, due to the displacement of the near-wall structures away from the wall and reducing the frictional drag. ${ }^{14-16}$

The influence of the addition of microbubbles on the coherent fluid structure has been commonly thought to be the key factor in the modification of the velocity fields and the wall shear stress. Nevertheless, uniformly dispersed microbubbles cannot modify the structure very well, as shown by our estimation above. It is necessary to investigate the two-way interactions between the microbubbles and flow structures, and further, the clustering of bubbles. The detailed distribution and motion of microbubbles, however, have not been discussed because of difficulties in the simultaneous measurement for both microbubble motion and the velocity field of the surrounding liquid phase. Furthermore, the measurement of the turbulent structures itself is still a challenging problem because of the complex three-dimensional characteristics of the flows.

To simplify the structure in order to investigate the effect of the addition of microbubbles on the coherent flow structure, Taylor-Couette flows ${ }^{17,18}$ that are flows between two concentric cylinders driven by their rotation have been adopted as platforms for experimental investigations. The major advantages of using Taylor-Couette flows for the evaluation are: (a) the system is closed, unlike those spatial-developing flows, for example, channel flows and boundary layers on flat plates, (b) the flow modes and transitions in the system can be controlled well, and (c) the system provides rich knowledge of single phase flows. ${ }^{19,20}$ Because of these advantages, so far, Taylor-Couette flows containing bubbles have been both experimentally and numerically investigated. ${ }^{13,21-27}$ Relatively large bubbles with $O(1 \mathrm{~mm})$ in diameter reduce the frictional drag by $20 \%$ by modifying the boundary layer in Taylor-Couette flows when bubbles are deformable. ${ }^{23,25}$ Bubble deformation or compressibility is also one of the factors in drag reduction as in spatially developing channel flows ${ }^{28,29}$ Non-deformable bubbles with $O(100 \mu \mathrm{m})$ in diameter, however, yield a drag reduction of $20 \%$ at most $^{26}$ even in a direct numerical simulation adopting a point-force coupling model. ${ }^{13}$ 
This phenomenon has been attributed to modifications of vortical structures; the axial elongation of the Taylor vortices by a localized buoyant force generated by preferentially accumulated bubbles, ${ }^{26}$ or global changes in the coherent Taylor vortex structure that reduce the Reynolds stress ${ }^{13}$ or switch the flow modes. ${ }^{27}$

This study aims to elucidate how the flow structure in a Taylor-Couette flow can be influenced by the addition of microbubbles with tiny volume concentrations (less than $0.1 \%$, i.e., a typical dilute two-phase condition). Modifications of the fluid properties in bulk, i.e., the density, viscosity, and extra energy, are of the same order as the void fraction, which are expected to be negligible. Countable numbers of basic flow elements, i.e., the basic shear flow (circular Couette flow), vortex array (Taylor vortices), and waves (azimuthally propagating waves), appear as flow instabilities in the laminar-transition flow regime in so called time-dependent flows. The number of flow elements increases in sequence of increasing the Reynolds number, and the variations in their magnitude can be described by the weakly nonlinear theory. The effects of the presence of microbubbles on the flow elements would be amplified in the periodic flow structure of the closed Taylor-Couette system and are easily observed. We carefully investigate the modification of the flow elements in the TaylorCouette system by using appropriate measurement equipment. The distribution of microbubbles on a cross section of the vortices is measured to evaluate the dynamic interaction of bubbles with coherent flow structures. Finally, we mention the effect of microbubbles on the flow transitions of the system, namely, a pseudo-decrease in the Reynolds number. The flow state subjected in this study is the beginning of the transition to turbulence and is not the fully developed turbulence considered in the frictional drag reduction problems. However, elucidating the interaction between microbubbles and the fundamental flow elements should provide important insights for understanding the drag reduction mechanisms.

\section{EXPERIMENTAL SYSTEMS}

\section{A. Experimental setup and definitions}

The experiments were conducted in the vertical Taylor-Couette system shown in Fig. 1. The rotating inner cylinder with $95.1 \pm 0.1 \mathrm{~mm}$ in radius $\left(R_{\mathrm{i}}\right)$ was made of carbon fiber reinforced plastic (CFRP); its surface was painted black, and it was driven by a stepper motor with 500 steps/revolution. The stationary outer cylinder with $105 \pm 0.1 \mathrm{~mm}$ in inner radius $\left(R_{\mathrm{o}}\right)$ was made of transparent Plexiglas to allow the visualization of the flows generated in the gap between the concentric cylinders. The cylinders were held by Plexiglas end plates and were set in a rectangular transparent water jacket for visualization and to maintain the temperature of the test fluid at $20 \pm 0.5^{\circ} \mathrm{C}$. The gap distance between the cylinders, $d$, and their height, $h$, were $9.9 \mathrm{~mm}$ and $197 \pm 0.5 \mathrm{~mm}$, respectively. The corresponding radius ratio of the cylinders, $\eta\left(=R_{\mathrm{i}} / R_{\mathrm{o}}\right)$, and the aspect ratio of the fluid layer, $\Gamma(=h / d)$, are 0.906 and 19.9, respectively. The Reynolds number of

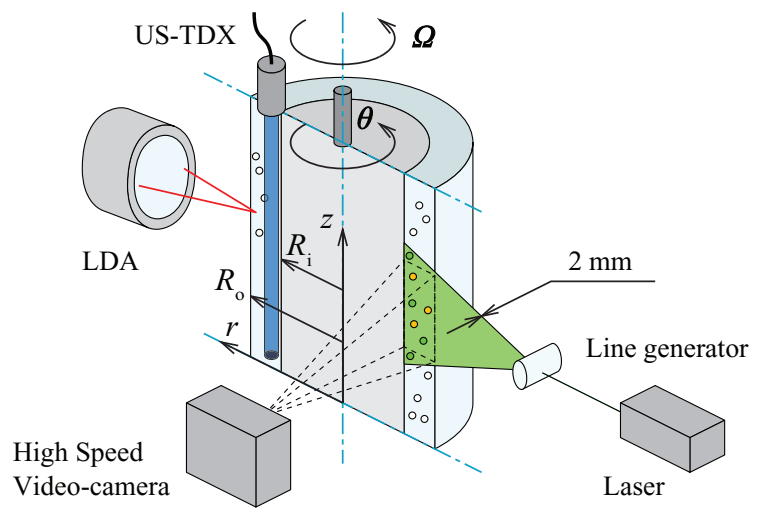

FIG. 1. Schematic outline of experimental setup. 


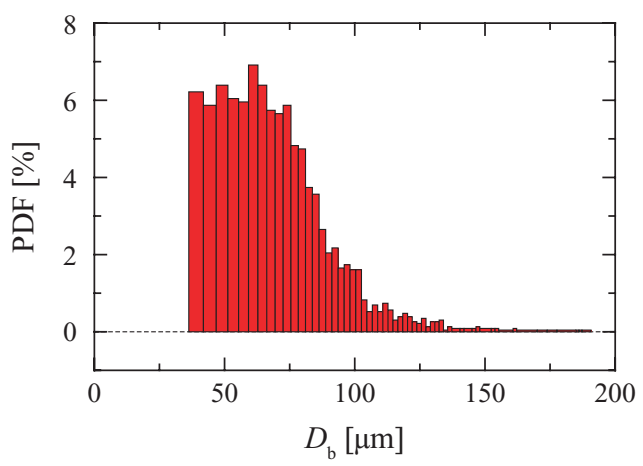

FIG. 2. Distribution of microbubble diameter.

the flow is defined as

$$
R e=\frac{U_{\text {wall }} d}{v}=\frac{2 \pi \Omega R_{\mathrm{i}}\left(R_{\mathrm{o}}-R_{\mathrm{i}}\right)}{v},
$$

where $U_{\text {wall }}, \Omega$, and $v$ are the inner wall velocity $\left(U_{\text {wall }}=2 \pi \Omega R_{\mathrm{i}}\right)$, rotation frequency, and kinematic viscosity of water, respectively, which is used as the test fluid. The reduced Reynolds number, $R e^{*}\left(=R e / R e_{\mathrm{c}}\right.$ ), is adjusted to $2 \leq R e^{*} \leq 9$ (i.e., $\left.269 \leq R e \leq 1210\right)$, where $R e_{\mathrm{c}}$ is the critical Reynolds number for the onset of the primary instability from the circular Couette flow (CCF) to Taylor vortex flow (TVF), $R e_{\mathrm{c}}=133.9$, which agrees rather well with the published value, $R e_{\mathrm{c}}=134.5 .^{30}$ In this range of the Reynolds number, the flow regimes correspond to wavy vortex flow (WVF, azimuthal waves superimposed on the Taylor vortices) and modulated wavy vortex flow (MWVF, in which the wave modulation appears on the azimuthal waves). ${ }^{19}$ In these experiments, first the rotation velocity of the inner cylinder was set at a value corresponding to the highest $R e$ with a fixed acceleration rate. Then, the velocity was decreased to smaller values to avoid the excitation of unstable flow modes while varying the $R e .^{31}$ The eigenvalues of the flow (the axial wavelength of the Taylor vortices, frequency of waves, number of azimuthal waves, and traveling velocity of waves) occurred within a certain range throughout all of the experiments.

Hydrogen bubbles with $O(10 \mu \mathrm{m})$ in diameter were generated by water electrolysis from a platinum wire with $100 \mu \mathrm{m}$ in diameter mounted at the bottom of the fluid layer (i.e., on the surface of the bottom end plate) and are dispersed into the fluid layer. The bubble diameter was measured by image analysis. Bubbles in an image are identified by giving a threshold value of their intensity. The object diameter of the bubbles is measured, and then, their probability density function (PDF) is calculated. The PDF of the bubble diameter is shown in Fig. 2. The mean bubble diameter was $64 \mu \mathrm{m}$, which is $1 / 6$ of the Kolmogorov length scale in the minimum $R e$ case of the two-phase condition and $1 / 3$ of the Kolmogorov scale in the maximum Re case. Here, the Kolmogorov length scale, $l$, is given as

$$
l=\left\{\frac{\pi v\left(R_{\mathrm{o}}^{2}-R_{\mathrm{i}}^{2}\right)}{1.45 U_{\text {wall }}} \frac{(1-\eta)^{\frac{7}{4}}}{(\operatorname{Re} \eta)^{\frac{3}{2}}}\right\}^{\frac{1}{4}},
$$

where $v, R_{\mathrm{i}}, R_{\mathrm{o}}, \eta$, and $U_{\text {wall }}$ are the kinematic viscosity of the test fluid, inner and outer cylinder radii, radius ratio of the cylinders, and inner cylinder wall velocity, respectively (see also the Appendix for the detailed derivation). The void fraction, $\alpha$, was estimated using the input power for electrolysis. The maximum value of $\alpha$ was smaller than $1.2 \times 10^{-4}$. The experimental conditions are listed in Table I.

\section{B. Measurement techniques}

Particle Tracking Velocimetry (PTV), Ultrasonic Velocity Profiling (UVP), and Laser Doppler Anemometer (LDA) were used selectively for measurements depending on their suitability for 
TABLE I. Experimental conditions.

\begin{tabular}{llc}
\hline \hline Contents & \multicolumn{1}{c}{ Symbols } & Values \\
\hline Inner cylinder radius & $R_{\mathrm{i}}$ & $95.1 \pm 0.1 \mathrm{~mm}$ \\
Outer cylinder radius & $R_{\mathrm{O}}$ & $105 \pm 0.1 \mathrm{~mm}$ \\
Gap distance & $d=R_{\mathrm{O}}-R_{\mathrm{i}}$ & $10 \mathrm{~mm}$ \\
Radius ratio & $\eta=R_{\mathrm{i}} / R_{\mathrm{O}}$ & 0.905 \\
Cylinder height & $h$ & $197 \pm 0.5 \mathrm{~mm}$ \\
Aspect ratio & $\Gamma=h / d$ & 19.9 \\
Kinematic viscosity & $v$ & $1.0 \times 10^{-6} \mathrm{~m}^{2} / \mathrm{s}$ \\
Density of test fluid & $\rho$ & $999.9 \mathrm{~kg} / \mathrm{m}^{3}$ \\
Mean void fraction & $\alpha$ & 0 to $1.2 \times 10^{-4}$ \\
\hline \hline
\end{tabular}

various purposes in experiments. The velocity distributions of the liquid phase and velocity vectors of the dispersed microbubbles on the radial-axial cross section were measured by image analysis consisting of PTV applying Laser Induced Fluorescence (LIF) using a single color-imaging video camera. This measurement method provides time series of the velocity vector field of the liquid phase flow, i.e., instantaneous velocity vectors and position vectors of the microbubbles at moments, namely, $\boldsymbol{u}\left[u_{r}(r, z, t), u_{z}(r, z, t)\right], \boldsymbol{u}_{\text {bubble }}\left[u_{r}\right.$ bubble $(j(t)), u_{z}$ bubble $\left.(j(t))\right]$, and $\boldsymbol{x}_{\text {bubble }}\left[r_{\text {bubble }}(j(t)), z_{\text {bubble }}(j(t))\right]$, where $(j(t))$ denotes the $j$ th bubble at time $t$. The number density of the velocity vectors for microbubbles, $N_{\text {bubble }}$, indicates the instantaneous local void fraction at a moment. Information on the color of the scattering light from the gas-liquid interface or from fluorescent particles in response to an incident laser sheet was used for the criteria to distinguish tracer particles and microbubbles. The details of the technique are described in Watamura et al. ${ }^{32}$ The measurement section, i.e., the $r-z$ cross section of the fluid layer in the middle of the axial direction, was illuminated by a laser sheet $(2 \mathrm{~W}$, $532 \mathrm{~nm}$ ) with $2 \mathrm{~mm}$ thickness. Resin particles with $15 \mu \mathrm{m}$ diameter containing LIF dye (EBM Co., Fluostar, specific gravity: 1.1, absorption wavelength: $550 \mathrm{~nm}$, emission wavelength: $580 \mathrm{~nm}$ ) were seeded as tracer particles that follow the flow. The Stokes number that represents the relative response time of the particles in the flow for a typical velocity fluctuation of the wave frequency, $f$, is defined as

$$
S t=\frac{\left(\rho_{\mathrm{p}}+\rho\right) D_{\mathrm{p}}^{2} f}{12 \mu}
$$

where $\rho_{\mathrm{p}}, \rho, D_{\mathrm{p}}$, and $\mu$ are the density of the particles, density of the test fluid, particle diameter, and viscosity of the test fluid, respectively. The Stokes number of the tracer particles was $S t \sim O\left(10^{-4}\right)$ and the volume concentration was $1 \times 10^{-5}$. Particles with color information were acquired by a color-imaging high-speed video camera (Photron Co., Fastcam MAX $120 \mathrm{KC}$ ) in a spatial range of $z=80 \mathrm{~mm}$ to $120 \mathrm{~mm}$ (i.e., $z^{*}(=z-h / 2)=-2 d$ to $2 d$, where $d$ is the gap distance of the fluid layer) with a spatial resolution corresponding to $39 \mu \mathrm{m} /$ pixel. The temporal resolution was $8 \mathrm{~ms}$ and the velocity resolution was $0.78 \mathrm{~mm} / \mathrm{s}$, respectively. Grid point relocation of the velocity vectors and interpolation for lacking velocity vectors at grid points were conducted to obtain the velocity vector distribution of the liquid phase. ${ }^{33}$ Then the stream function of the flow field was calculated to determine the global motion of the oscillating Taylor vortex array appearing in the fluid layer. ${ }^{18,27}$

The velocity distributions of the axial component were also measured by $\mathrm{UVP}^{34}$ as well as image analysis to measure the power of the axial velocity fluctuations and to determine the flow mode of the Taylor-Couette flow. UVP can record instantaneous velocity profile information for much longer than PTV can do, thanks to real-time data acquisition in UVP. It has, therefore, a great advantage for obtaining the temporal statistics of velocity fluctuations. Resin particles with $60 \mu \mathrm{m}$ in mean diameter were seeded as tracer particles in a volume concentration of $5 \times 10^{-4}$, where $S t \sim O\left(10^{-3}\right)$. An ultrasonic transducer (US-TDX) was mounted on the top end wall near the rotating inner cylinder (see Fig. 1), being parallel to the rotational axis. A time series of instantaneous velocity profile at $\left(r-R_{\mathrm{i}}\right) / d=0.25$ was measured as a function of the axial position and time, namely, $u_{z}(z, t)$ along the inner cylinder. The measurement volume of each point had the shape of a disk 
with $5 \mathrm{~mm}$ diameter (for the radial and tangential directions) and $0.70 \mathrm{~mm}$ thickness (for the axial direction). The sampling period was $72 \mathrm{~ms}$ and the velocity resolution was $0.39 \mathrm{~mm} / \mathrm{s}$, respectively. The velocity profiles were measured from $z=50 \mathrm{~mm}$ to $150 \mathrm{~mm}$ (i.e., $z^{*}=-5 d$ to $5 d$ ) to eliminate the end wall effect as much as possible.

The mean tangential velocity component, i.e., the main flow in a Taylor-Couette system, was measured by the LDA using a $5 \mathrm{~mW}$ diode laser. Because PTV and UVP are not suitable for measuring the tangential velocity component in this configuration, LDA measurement was adopted as a practical, conventional, and noninvasive method of measuring the velocity component in the narrow fluid layer. The measurement point was scanned on a meridional plane using a three-axis traverse system with a positioning accuracy of $0.1 \mathrm{~mm}$. The measurements were conducted from $z=70 \mathrm{~mm}$ to $130 \mathrm{~mm}$, i.e., $z^{*}=-3 d$ to $3 d$, at $4 \mathrm{~mm}$ intervals. The LDA measurements yielded a time series of the tangential velocity $u_{\theta}(r, z, t)$ measured spatially at each point. The resin particles used for UVP measurement were also seeded as the flow tracers with the same concentration as that for UVP. The size of the measurement volume was $450 \mu \mathrm{m}$ in the radial direction and $60 \mu \mathrm{m}$ in the tangential and axial directions, respectively. The sampling period was $100 \mathrm{~ms}$ and the maximum velocity resolution was $2.3 \mathrm{~mm} / \mathrm{s}$, respectively. To minimize uncertainties due to the curvature of the outer cylinder, the position and beam angle were carefully calibrated.

\section{DETERMINATION OF FLOW MODE}

Since Couette's ${ }^{17}$ and Taylor's ${ }^{18}$ studies, the flow modes in supercritical Taylor-Couette flows have been well investigated and were categorized as a function of the Reynolds number on the basis of the rotation speed of the cylinders. ${ }^{19}$ For quantitative understanding of the two-phase states, the flow modes in both the single phase and two-phase conditions are determined in our experimental system. Axially aligned Taylor vortices oscillate in the axial direction, and the oscillation (waviness of the vortex tube) propagates in the azimuthal direction with the basic frequency of the WVF mode. With increasing Reynolds number, the modulation wave appears as an additional azimuthal wave in MWVF. The power of the velocity fluctuation of the basic wave, $\tilde{u}_{z}^{2}\left(f_{\mathrm{w}}\right)$, or the modulation wave, $\tilde{u}_{z}^{2}\left(f_{\mathrm{m}}\right)$, is obtained from a power spectrum of the spatio-temporal distribution of the axial velocity component measured by UVP (e.g., Takeda et al. ${ }^{31,35}$ ). The power of the waves is measured at various Reynolds numbers, and their spatial averages, $\left\langle\tilde{u}_{z}{ }^{2}\right\rangle$, are shown in Fig. 3, where angle brackets, \langle\rangle , indicate the spatial average from $5 d$ to $15 d$. The error bars in the figure show the magnitude of the standard deviations of the ensemble average in several runs of experiments. The power of the basic wave increases until $R e^{*} \sim 5$. The modulation wave, GS mode, ${ }^{36}$ appears for $R e^{*}>5$, and its power increases. Because of the appearance of the modulation wave, the power of the basic wave decreases and is decentralized into linear combination components. The waviness, i.e., the axial oscillation of the Taylor vortex array propagating in the azimuthal direction, in a Taylor-Couette system is related to the growth of instability in the out-flow region, in which a radial jet extends from near the inner cylinder to the outer one between a pair of Taylor vortices. ${ }^{37-40}$ According to the theory of weakly nonlinear growth of perturbations, ${ }^{41,42}$ the amplitude of grown perturbation, $\tilde{u}$, increases proportionally to the square root of Reynolds number as

$$
\tilde{u}=C \sqrt{R e-R e_{\text {critial }}}
$$

where $R e_{\text {critical }}$ and $C$ are the critical Reynolds number for the appearance of instability and growth rate of the wave power, respectively. Consequently, the fluctuation power of the wave, $\tilde{u}_{z}^{2}$, increases as a linear function of the Reynolds number, $R e$. The lines in Fig. 3 are least square approximation lines following the basic and modulation powers. The critical Reynolds numbers for WVF, $R e_{\mathrm{w}}$ and MWVF, $R e_{\mathrm{m}}$ are determined as the cross points between the lines and the $R e$-axis. The growth rate as a function of the Reynolds number in the basic wave, $C_{\mathrm{w}}$, and modulation wave, $C_{\mathrm{m}}$, is determined from the gradient of the lines in Fig. 3. The critical Reynolds numbers and growth rates are listed in Table II. The critical points for the onset of each mode are delayed by the addition of microbubbles. The growth rate of the wave amplitudes are also modified by the addition of the microbubbles: The 

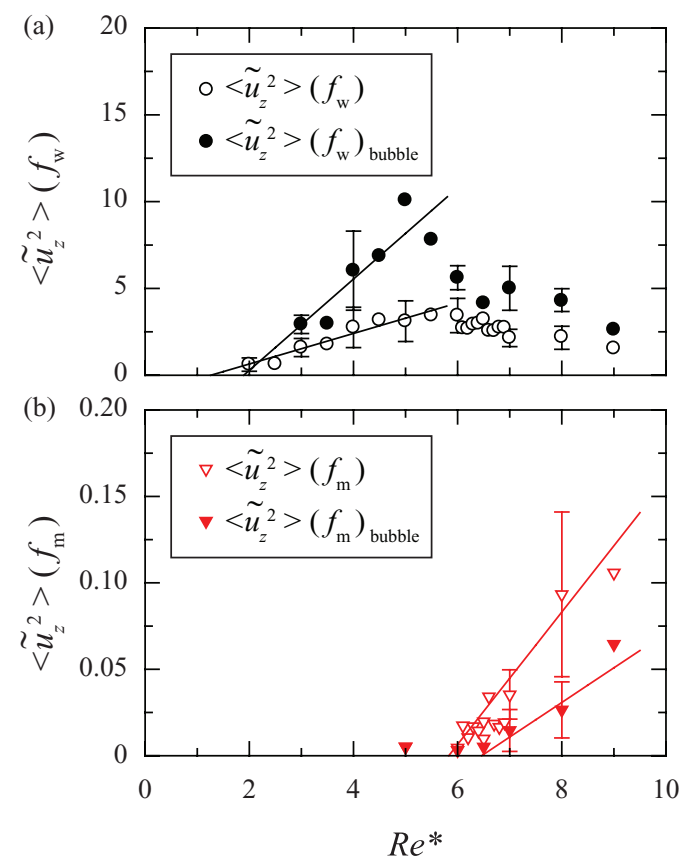

FIG. 3. Power of axial velocity fluctuations of (a) basic wave $\left\langle\tilde{u}_{z}{ }^{2}\right\rangle\left(f_{\mathrm{w}}\right)$ and (b) modulation wave $\left\langle\tilde{u}_{z}{ }^{2}\right\rangle\left(f_{\mathrm{m}}\right)$ versus normalized Reynolds number $R e^{*}$ in single phase (open symbols) and two-phase conditions (closed symbols, $\alpha=6 \times 10^{-5}$ ). The lines represent the least square approximation lines following the basic and modulation powers, and the error bars indicate the magnitude of the standard deviations of ensemble average in runs of experiments.

growth rate of the basic wave amplitude is increased and that of the modulation wave amplitude is decreased.

\section{RESULTS}

\section{A. Bubble distribution}

An instantaneous distribution of microbubbles in an $r-z$ cross section of the wavy Taylor vortex structure is shown in Fig. 4, where the reduced Reynolds number $R e^{*}=8$ (MWVF) and the void fraction $\alpha=6 \times 10^{-5}$. The panels at the bottom left ((b)-(d)) and bottom right ((e)-(g)) show the results obtained at the crest and trough of the azimuthal wave on the vortices illustrated in Fig. 4(a), respectively. The figures in each panel represent the velocity vector field of the liquid phase ((b) and (e)), corresponding stream function ((c) and (f)), and velocity vectors of microbubbles ((d) and $(\mathrm{g})$ ), respectively. At the crest (left panel), microbubbles are accumulated in the axial flow that connects adjacent vortices. In contrast, microbubbles do not show any typical pattern of ascent at the trough (right panel). Taylor vortex structures are represented by stream function, $\psi$, in Figs. 4(c) and 4(f). The colors indicate the rotation direction; reddish and bluish colors correspond

TABLE II. Critical Reynolds numbers and growth rates of wave powers; $R e_{\mathrm{c}}, R e_{\mathrm{w}}$, and $R e_{\mathrm{m}}$ are the critical Reynolds number for the onset of instability from CCF to TVF, TVF to WVF, and WVF to MWVF, respectively. $C_{\mathrm{w}}$ and $C_{\mathrm{m}}$ are the growth rates of the basic and modulation wave, respectively, as a function of the $R e^{*}$.

\begin{tabular}{lllc}
\hline \hline Flow mode & \multicolumn{1}{c}{ Valuables } & Single phase & Two-phase $\left(\alpha=6 \times 10^{-5}\right)$ \\
\hline TVF to WVF & $\operatorname{Re}^{*}{ }_{\mathrm{W}}\left(=R e_{\mathrm{w}} / R e_{\mathrm{c}}\right)$ & 1.26 & 1.89 \\
& $C_{\mathrm{w}}{ }^{2}$ & 0.878 & 2.63 \\
WVF to MWVF & $R e^{*}{ }_{\mathrm{m}}\left(=R e_{\mathrm{m}} / R e_{\mathrm{c}}\right)$ & 5.83 & 6.46 \\
& $C_{\mathrm{m}}{ }^{2}$ & $3.83 \times 10^{-2}$ & $2.00 \times 10^{-2}$ \\
\hline \hline
\end{tabular}


(a)
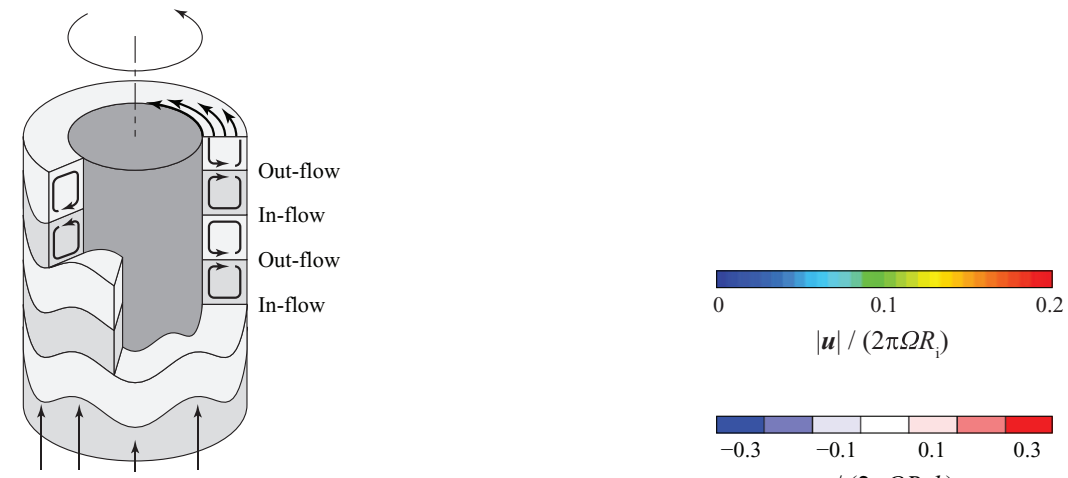

Trough Crest Trough Crest

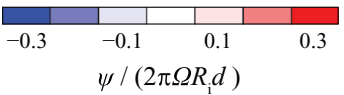

(b)

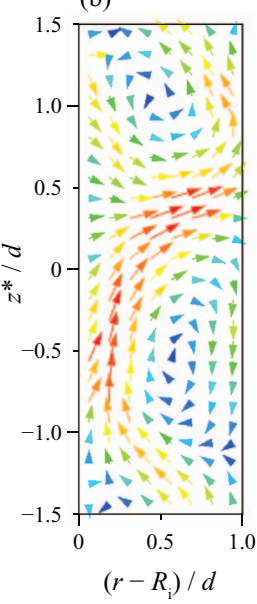

(c)

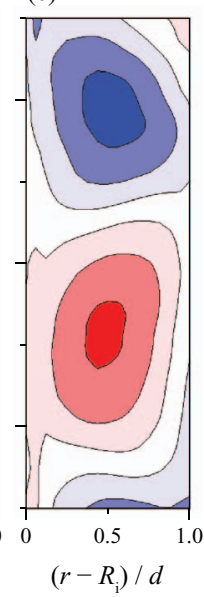

(d)

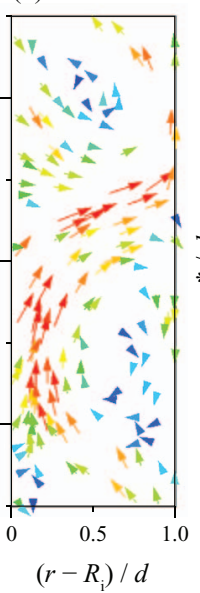

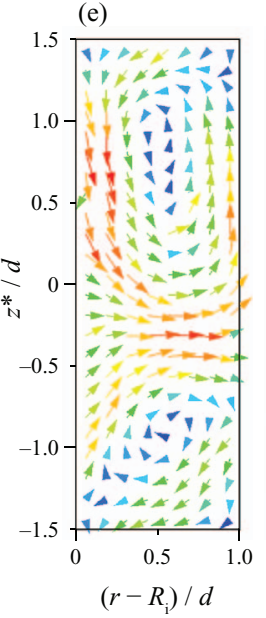

(f)

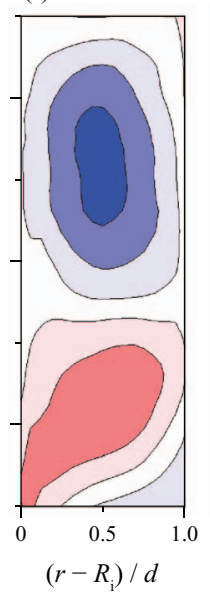

(g)

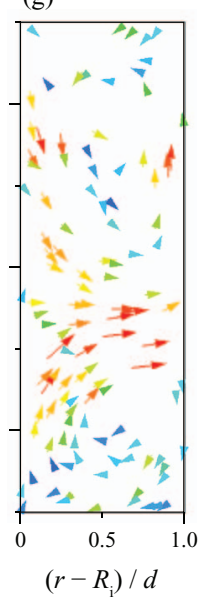

FIG. 4. (a) Axially oscillating Taylor vortices appearing in the fluid layer, (b) and (e) velocity vector field of liquid phase, (c) and (f) corresponding stream function, and (d) and (g) velocity vector of microbubbles at $R e^{*}=8$, where (b)-(d) and (e) $-(\mathrm{g})$ indicate the results obtained at the crest and trough of the wavy motion, respectively.

to clockwise $(\mathrm{CW})$ and counter clockwise $(\mathrm{CCW})$ rotation, respectively. The axial size of the Taylor vortices (axial wavelength) is unchanged by the addition of microbubbles relative to that in the single phase state. In Fig. 5, the time series of the stream function at the center line of the fluid layer, $\left(r-R_{\mathrm{i}}\right) / d=0.5$, shows a pair of axially oscillating torus vortices. Elongation in the axial direction, the advection of the vortex tube upward, or changes in the flow mode (torus/spiral) do not appear with the addition of bubbles, unlike the results of previous studies in which larger bubbles with $1 \mathrm{~mm}^{22}$ or $600 \mu \mathrm{m}^{26,27}$ mean diameter (so called milli-bubbles or sub-milli-bubbles) were added. Other eigenvalues of the Taylor-Couette flow, i.e., the frequency of the basic azimuthal waves, $f_{\mathrm{w}}$, its modulation frequency, $f_{\mathrm{m}}$, the wavenumber of the basic azimuthal wave, and the traveling velocity of the waves, were also unchanged. The presence of bubbles has no effect on the basic flow structure qualitatively (also mentioned by Murai et al. ${ }^{4}$ and Xu et al. ${ }^{14}$ ). This could be expected, since the variations in the properties of the fluids containing a dispersed phase, i.e., the density and viscosity in bulk, are of the same order as the void fraction, which is negligible in this experimental condition, $\alpha<1.2 \times 10^{-4}$. Quantitative analyses of the modification of the flow structure are presented in Sec. IV B.

Figure 6 shows (a) the positions of the vortex cores, defined as the position at which $\psi$ takes the maximum or minimum value, $\left.z\right|_{\psi_{\text {max min }}}$, where blue circles indicate the core positions of CCW rotating vortices and red circles indicate the core positions of $\mathrm{CW}$ rotating vortices, (b) the spatially averaged axial velocity of bubbles in the measurement plane $\left\langle u_{z \text { bubble }}\right\rangle$, and (c) the number density of bubbles in the measurement plane. The spatio-temporal average of the rising velocity of bubbles 


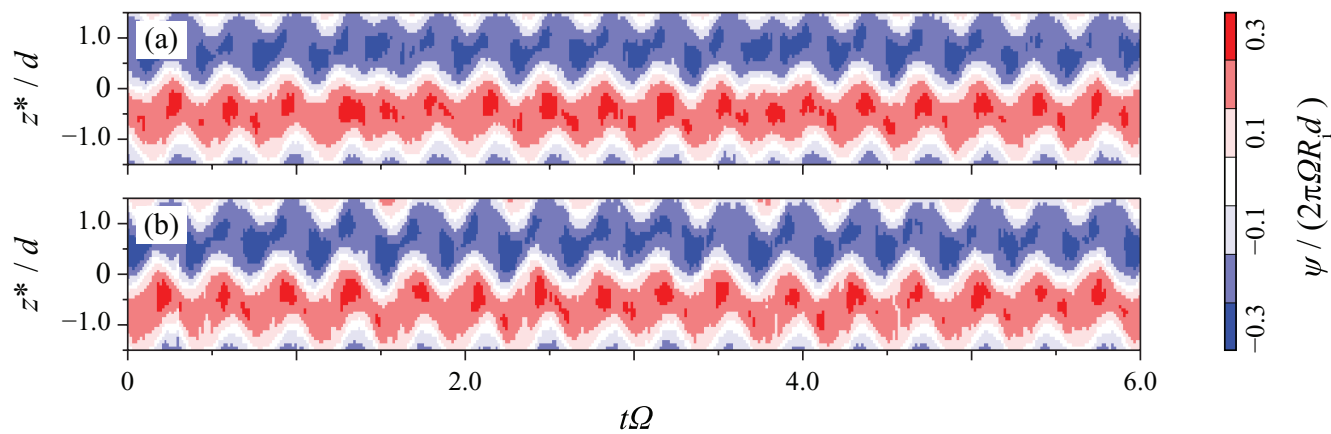

FIG. 5. Time series of stream function at the center of the fluid layer, $\left(r-R_{\mathrm{i}}\right) / d=0.5$, obtained at $R e^{*}=8$ : (a) single phase flow and (b) two-phase flow $\left(\alpha=6 \times 10^{-5}\right)$.

decreases with increasing the Reynolds number, $R e ;\left\langle\overline{u_{z \text { bubble }}}\right\rangle / U_{\mathrm{St}}=1.1$ at $R e^{*}=3$ and $\left\langle\overline{u_{z \text { bubble }}}\right\rangle / U_{\mathrm{St}}$ $=0.79$ at $R e^{*}=9$, where the overline, "“-", indicates the time average, and $U_{\mathrm{St}}$ is the Stokes rising velocity given by

$$
U_{\mathrm{St}}=\frac{\rho g D_{\mathrm{b}}^{2}}{12 \mu}
$$

where $\rho, \mu, g$, and $D_{\mathrm{b}}$ are the density and viscosity of the test fluid, gravitational acceleration, and mean bubble diameter, respectively. The dashed line in Fig. 6(b) indicates the spatio-temporal averaged value, $\left\langle\overline{u_{z \text { bubble }}}\right\rangle / U_{\mathrm{St}}=0.98$ at $R e^{*}=8$. Bubbles rise upward globally at the crests and descend at the troughs of the azimuthal wave. Further, the number density of bubbles increases at the crests and decreases at the troughs. This indicates that bubbles form an organized fluctuation of the void fraction, i.e., a voidage wave, in the azimuthal direction. The cross-correlation between the axial position of the vortex core and the number of detected bubbles that shows the relationship between the axial oscillation of the azimuthal wave and the voidage wave is 0.91 . Thus, it can be said that the microbubbly voidage waves that are highly synchronized with the azimuthal wave are generated in the flow field. This would be caused by a global (fluid layer scale) effect appearing as the result of mutual interaction between the bubble distribution and flow structure of the surrounding
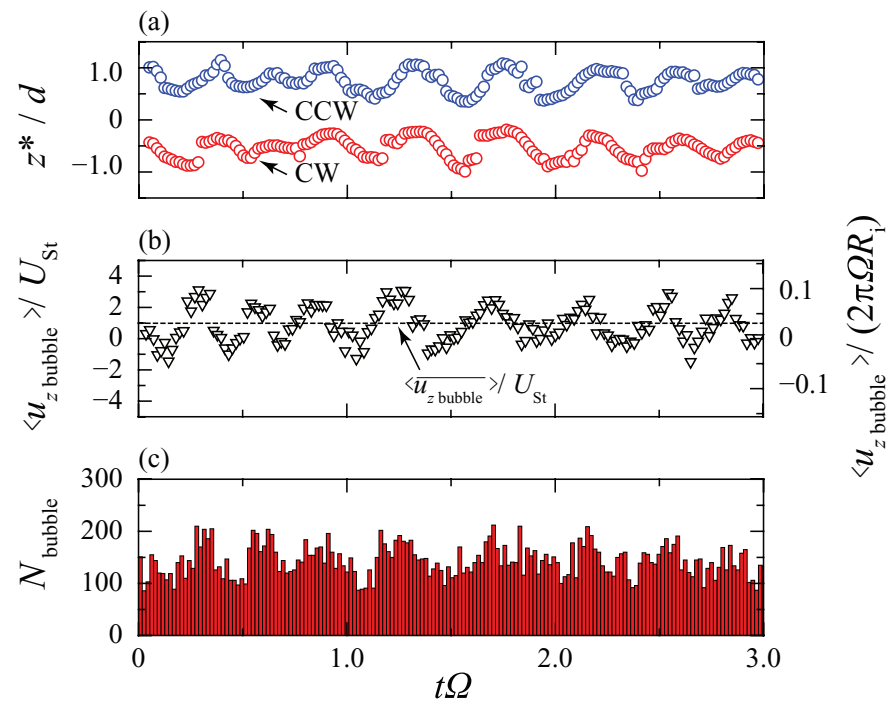

FIG. 6. Time series of (a) axial position of vortex core, where blue and red indicate counter clock wise (CCW) and clock wise (CW) rotation vortices, respectively, (b) spatially averaged axial velocity of microbubbles normalized by Stokes rising velocity of the microbubble, $U_{\mathrm{St}}$, and the inner cylinder wall velocity, $2 \pi \Omega R_{\mathrm{i}}$, and (c) number of microbubbles. 
TABLE III. Variations in typical quantities of flow elements in Taylor-Couette flow at two void fractions.

\begin{tabular}{lccc}
\hline \hline & $\alpha=6 \times 10^{-5}(\%)$ & $\alpha=1.2 \times 10^{-4}(\%)$ & Measurement instruments \\
\hline$\left\langle\tilde{u}_{z}^{2}\right\rangle\left(f_{\mathrm{w}}\right)$ & +94 & +122 & UVP, PTV \\
$\left\langle\tilde{u}_{z}^{2}\right\rangle\left(f_{\mathrm{m}}\right)$ & -72 & -78 & UVP, PTV \\
$\left.\tilde{z}\right|_{\psi_{\max , \min }}\left(f_{\mathrm{w}}\right)$ & +7 & +51 & PTV \\
$\tilde{\psi}_{\max , \min }\left(f_{\mathrm{w}}\right)$ & +13 & +55 & PTV \\
$\tilde{\psi}_{\max , \min }\left(2 f_{\mathrm{w}}\right)$ & -5 & -13 & PTV \\
$-\left.\frac{\partial}{\partial r}\left\langle\overline{u_{\theta}}\right\rangle\right|_{r=R_{\mathrm{i}}}$ & N/A & -8 & LDA \\
$-\left.\frac{\partial}{\partial r}\left\langle\overline{u_{\theta}}\right\rangle\right|_{r=R_{\mathrm{o}}}$ & N/A & -11 & LDA \\
$\left\langle\tilde{u}_{\theta}\right\rangle\left(f_{\mathrm{w}}\right)$ & N/A & -52 & LDA \\
\hline \hline
\end{tabular}

liquid phase. Moreover, the fluctuation amplitude of the Taylor vortex tube in the axial direction, $\left.\tilde{z}\right|_{\psi_{\max , \min }}$, is greater in the two-phase conditions (Table III). The oscillation amplitude of the vortex tubes generally decreases with increasing Reynolds number. ${ }^{43}$ The resulting effect shows that the addition of microbubbles to the flows enhances the basic wave oscillation of the Taylor vortex tubes and modifies the flow state into that obtained with lower Reynolds number.

Shiomi et al., ${ }^{21}$ Djeridi et al. ${ }^{22}$ and Murai et al. ${ }^{26}$ have experimentally investigated the distribution of bubbles in a vertical Taylor-Couette system. Murai et al. ${ }^{26}$ concluded that the bubble motions captured in the vortical structure are governed by the Froude number, i.e., the centrifugal acceleration relative to the gravity/buoyancy, defined as

$$
F r=\frac{U_{\text {wall }}}{\sqrt{g R_{\mathrm{i}}}},
$$

where $U_{\text {wall }}, g$, and $R_{\mathrm{i}}$ are the inner wall velocity $\left(U_{\text {wall }}=2 \pi \Omega R_{\mathrm{i}}\right)$, gravity acceleration, and radius of the inner cylinder, respectively. Bubbles in the Taylor-Couette flows are trapped into the core of the Taylor vortices when $F r$ is greater than 0.6. Under our experimental conditions, the maximum Froude number $F r$ is 0.1 and bubbles would not be trapped into the vortical structure. Because of the considerable slip velocity between the bubbles and the liquid phase flows, bubbles with $O(1 \mathrm{~mm})$ in diameter are captured in the cores of the vortices at the crests and troughs alternately, which are the local minima of the pressure, in the relatively weak wavy Taylor vortex flow. ${ }^{24}$ The bubbles, however, migrate and rise in the axial flow when they are ejected from the core of the Taylor vortices in the relatively strong wavy flow mode. ${ }^{24}$ Our observation agrees well with this expectation based on the governing relation of the force balance, $F r$. The organized azimuthal distributions of bubbles that resemble those in Fig. 6 have also been observed in the previous studies by Murai et al. ${ }^{26}$ and Yoshida et al. ${ }^{27}$ which used relatively small bubbles with $O(100 \mu \mathrm{m})$ diameter. The mechanisms that generate the organized inhomogeneous bubble distribution, however, have not been discussed and are still unknown. Fluid particles experience periodic accelerations and pressure fluctuations in the waviness of $\mathrm{WVF}^{44}$ in the Lagrangian-frame. The voidage wave, i.e., the fluctuation in the number density of microbubbles, in the azimuthal direction might be related to the integration of the drift due to the small but unignorable slip velocity in the periodically accelerated wavy flow. Because the rising velocity of microbubbles is relatively small compared to the mean azimuthal velocity of the fluid, about $40 \%$ of the inner wall velocity, ${ }^{45}$ microbubbles form a preferential concentration pattern in the long-time scale of travel in the azimuthal wavy flow patterns.

\section{B. Modification of secondary flow mode}

Figure 7 shows spatially averaged power spectra of the axial velocity fluctuation $\left\langle\tilde{u}_{z}^{2}\right\rangle$ obtained by UVP for various $R e^{*}$ values. The figure panels on the left represent the single-phase condition and those on the right represent the two-phase condition, $\alpha=6 \times 10^{-5}$. The frequency of the basic wave, $f_{\mathrm{w}}$, and its modulation, $f_{\mathrm{m}}$, are unchanged by the addition of microbubbles under our experimental conditions. The power of the basic wave fluctuation increases with adding microbubbles, whereas that of the modulation wave decreases in the MWVF (Figs. 7(b) and 7(c)). In any case, peak frequencies 
(a)

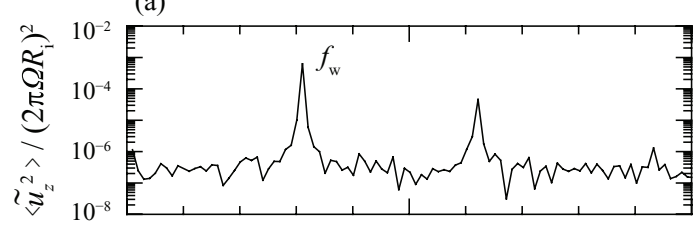

(b)

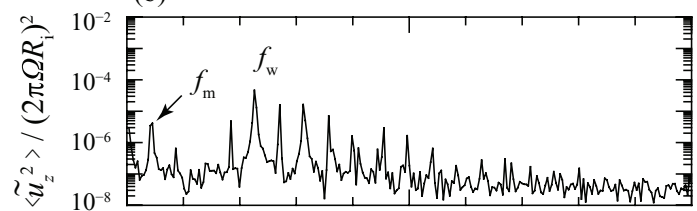

(c)

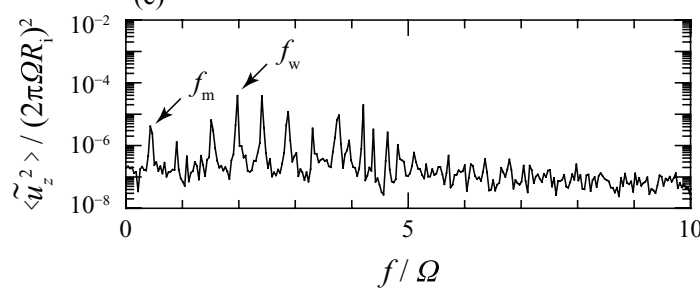

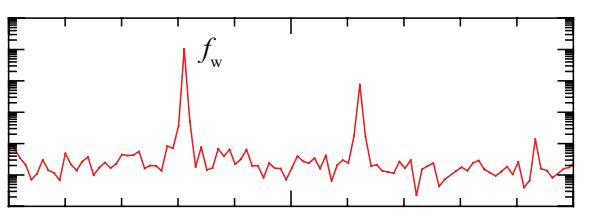
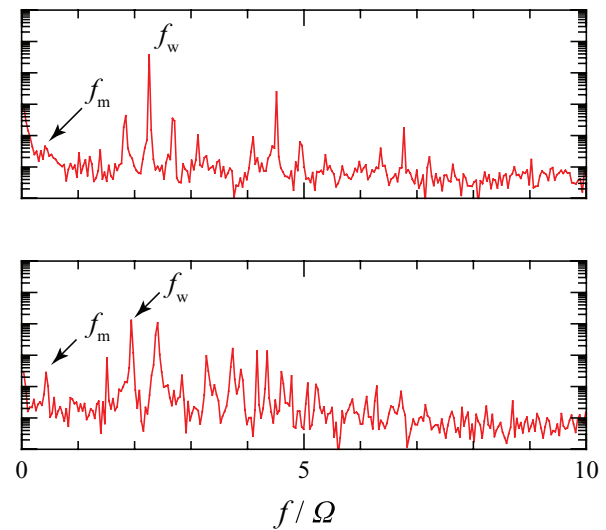

FIG. 7. Comparison of power spectra of the axial velocity fluctuation, $\left\langle\tilde{u}_{z}{ }^{2}\right\rangle$, normalized by the inner cylinder wall velocity, $2 \pi \Omega R_{\mathrm{i}}$, at (a) $R e^{*}=3$, (b) $R e^{*}=8$, and (c) $R e^{*}=9$. Panels on the left represent single phase flow, whereas panels on the right represent two-phase flow $\left(\alpha=6 \times 10^{-5}\right) ; f_{\mathrm{w}}$ and $f_{\mathrm{m}}$ represent the basic and modulation frequencies, respectively.

in the spectra can be described as linear combinations of $f_{\mathrm{w}}$ and $f_{\mathrm{m}}$, hence, these results conclude that the addition of microbubbles does not create any additional frequency components of the oscillating flows. Variations in the powers of the components of MWVF, $f_{\mathrm{w}}$ and $f_{\mathrm{m}}$, with various void fractions, $\alpha$, are presented in Fig. 8, where the background component (BG) represents the minimum power between the frequency components of $f_{\mathrm{w}}$ and $f_{\mathrm{m}}$. Open symbols in the figure represent UVP data, and solid symbols represent PTV data, $\left\langle\tilde{u}_{z}{ }^{2}\right\rangle$ at $\left(r-R_{\mathrm{i}}\right) / d=0.25$, respectively. The error bars indicate the magnitudes of the standard deviation of the ensemble average in several runs of experiments. The powers of waves are determined by Fourier transform of the axial velocity fluctuation with a finite measurement time length, which depends on the measurement equipment. Owing to this experimental limitation of the measurement time (mentioned in Sec. II B), the magnitudes of the powers of the waves obtained from PTV and UVP differ in Fig. 8(b). The modifications of the powers of the basic and modulation waves show the same tendency of the variation. The power of the basic wave increases with increasing $\alpha$ for all $R e^{*}$. In contrast, the power of the modulation wave decreases with increasing $\alpha$. Further, the peak representing the modulation component is buried in the background components, particularly for $\alpha>3 \times 10^{-5}$ at $R e^{*}=7$. These results suggest that the addition of microbubbles enhances the basic wave and reduces the modulation wave. As shown in Fig. 3, the development of the flow transition provides enhancement of the modulation component and decay of the basic wave. The addition of microbubbles, however, prevents the flow transition in the variations of the oscillating wave power. In each graph in Fig. 8, unstable changes appeared at a very low void fraction of microbubbles, less than $\alpha<2 \times 10^{-5}$. One of the considerable reasons is that the number of microbubbles is very few in this range of the void fraction. Hence, they cannot be distributed equally to arranged Taylor vortices. This is an inevitable but common effect of dilute dispersive multiphase flows being discussed like a continuum.

The qualitative expression of the stream function distribution in the $r$-z plane (Figs. 4(c) and 4(f)) and the magnitude of the stream function, which is associated with the instantaneous strength of Taylor vortices (Fig. 5), also do not indicate significant modifications of the Taylor vortices by adding microbubbles. The fluctuations of the values at the center of the oscillating Taylor vortices, i.e., the maximum or minimum values of the stream function, $\tilde{\psi}^{2}$ max, min, in the single phase and two-phase cases are shown in Fig. 9. The frequencies of the fluctuations are also unchanged; the 

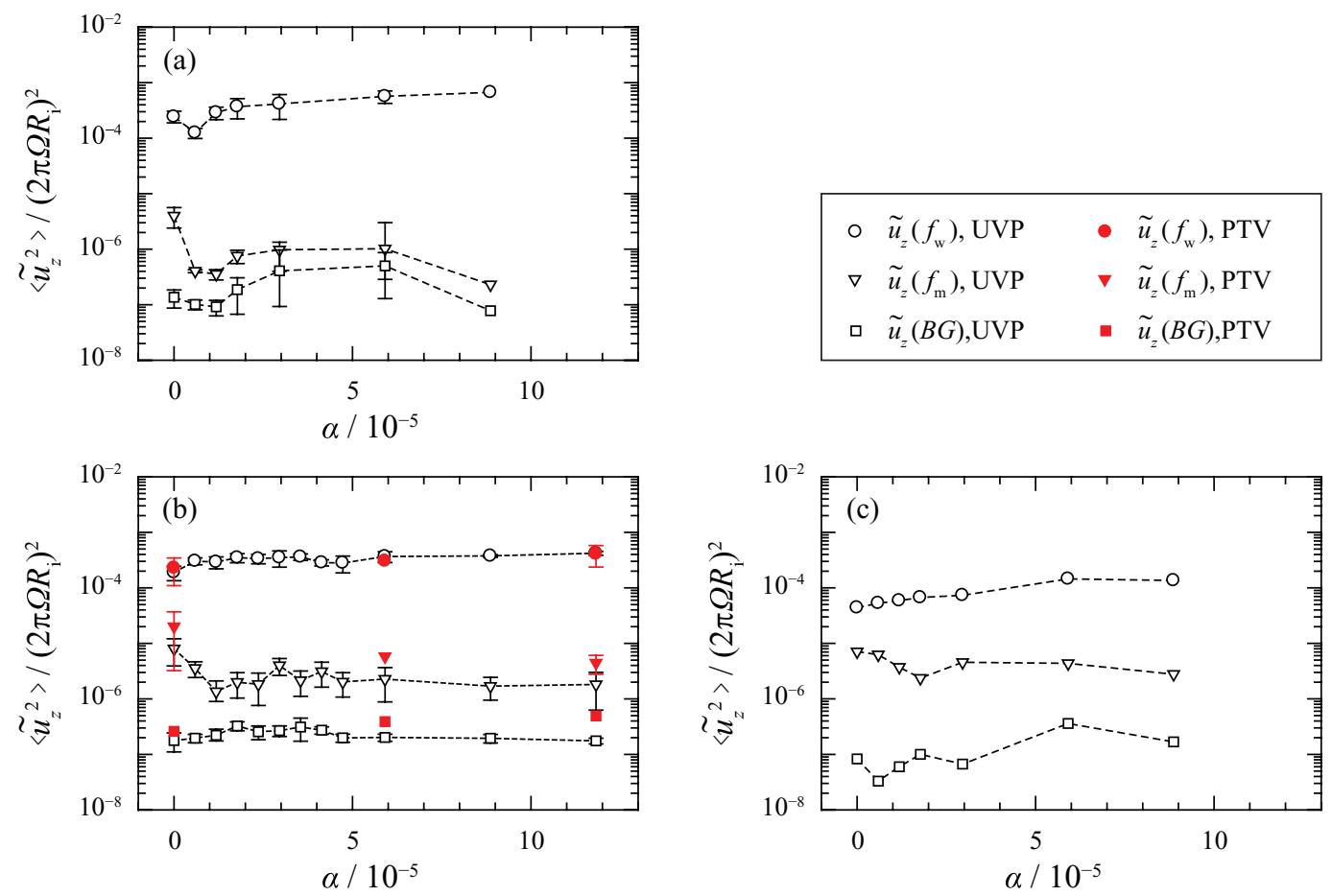

FIG. 8. Power of the basic wave, $\left\langle\tilde{u}_{z}\right\rangle\left(f_{\mathrm{w}}\right)$, modulation wave, $\left\langle\tilde{u}_{z}\right\rangle\left(f_{\mathrm{m}}\right)$, and background component, $\left\langle\tilde{u}_{z}\right\rangle(B G)$, at $\left(r-R_{\mathrm{i}}\right) / d=0.25$ versus the void fraction, $\alpha$, obtained by UVP (open symbols) and PTV (solid symbols): (a) $R e^{*}=7$, (b) $R e^{*}=8$, and (c) $R e^{*}=9$.
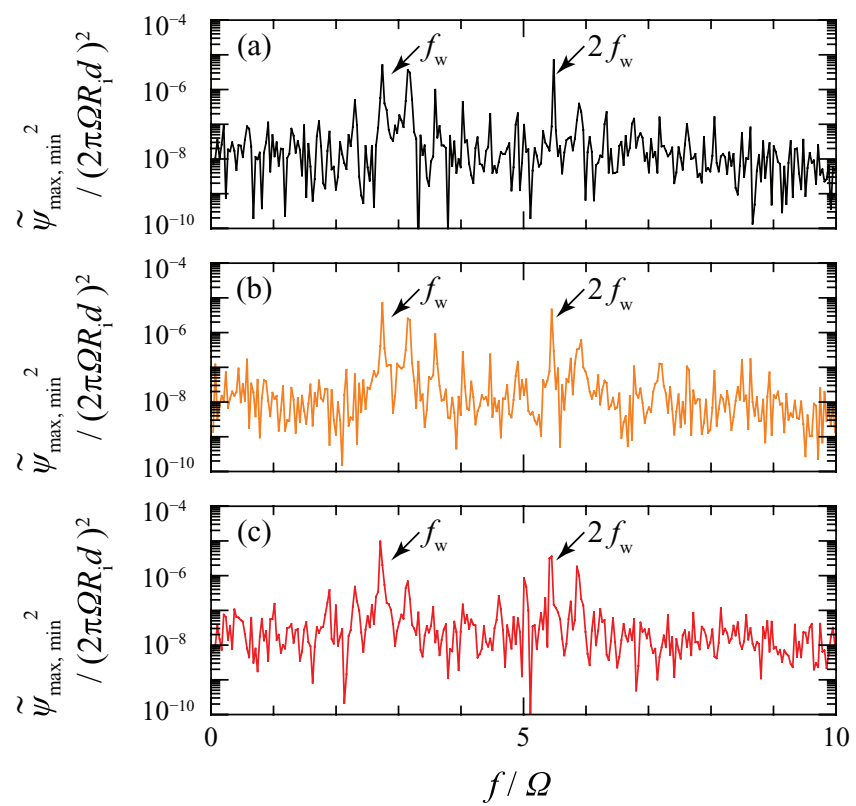

FIG. 9. Power spectra of the fluctuation in the stream function at the center of the vortices, $\tilde{\psi}_{\max },{ }_{\min },{ }^{2}$ at $R e^{*}=8$ : (a) single phase flow, (b) two-phase flow at $\alpha=6 \times 10^{-5}$, and (c) at $\alpha=1.2 \times 10^{-4}$, respectively. 
fluctuation powers, however, indicate an increase at the basic frequency, $f_{\mathrm{w}}$, and a decrease at twice the basic frequency, $2 f_{\mathrm{w}}$ (Table III). The fluctuation power corresponding to $2 f_{\mathrm{w}}$ increases with the nonlinear development of the waviness with increasing $R e$. This is the selective intensification of the vortex strength at the crest of the wave by preferentially rising bubbles (Figs. 4 and 6), which also appears in the velocity profiles (Figs. 7 and 8).

The values of the power for the basic wave, $f_{\mathrm{w}}$, and the modulation component, $f_{\mathrm{m}}$, in a twophase condition, $\alpha=1.2 \times 10^{-4}$, at $R e^{*}=8$ are comparable with those in the single phase condition at $R e^{*}=6.5$, i.e., the maximum modification (equivalent to a $20 \%$ decrease) of the relative Reynolds number (Fig. 3). The microbubbles seem to have little effect on the wave power at $R e^{*}=9$ in Figs. 7(c) and 8(c) compared to that at $R e^{*}=8$ in Figs. 7(b) and 8(b). The maximum influence on the intensification of the basic wave power appears at $R e^{*}=5$ in Fig. 3. These phenomena suggest that the influence of microbubbles on the waviness in flow is selective but would produce the optimum conditions for preventing the flow transition. The vortical structure of the Taylor vortex array in the $r-z$ cross section is maintained even in the two-phase conditions (see Fig. 4). The waviness of the vortex tube, however, is significantly modified by the selective motion of microbubbles conforming to the basic wave. Bubbles in this Taylor-Couette flow are preferentially distributed at the crest of the basic wave in the azimuthal direction, and the distribution may play a key role in the enhancement of the basic wave. Thus, the basic wave enhancement has the greatest effect at $R e^{*}=5$, which is just below the onset of wave modulation.

\section{Reduction of azimuthal shear}

The time-averaged distribution of the azimuthal velocity component is shown as contours in Fig. 10 for (a) single phase and (b) two-phase conditions with a void fraction of $\alpha=1.2 \times 10^{-4}$. Whiteness and blackness represent high and low velocities, respectively. An out-flow region in which the azimuthal jet carries the high azimuthal momentum outward exists around $z^{*}=0$ in both the single and two-phase conditions. The mean azimuthal flow structure remained in the twophase case, because the mean rising velocity of microbubbles is smaller compared to the inner wall velocity (see Fig. 6(b)). The high-velocity regions are expanded at the upper region of the out-flow

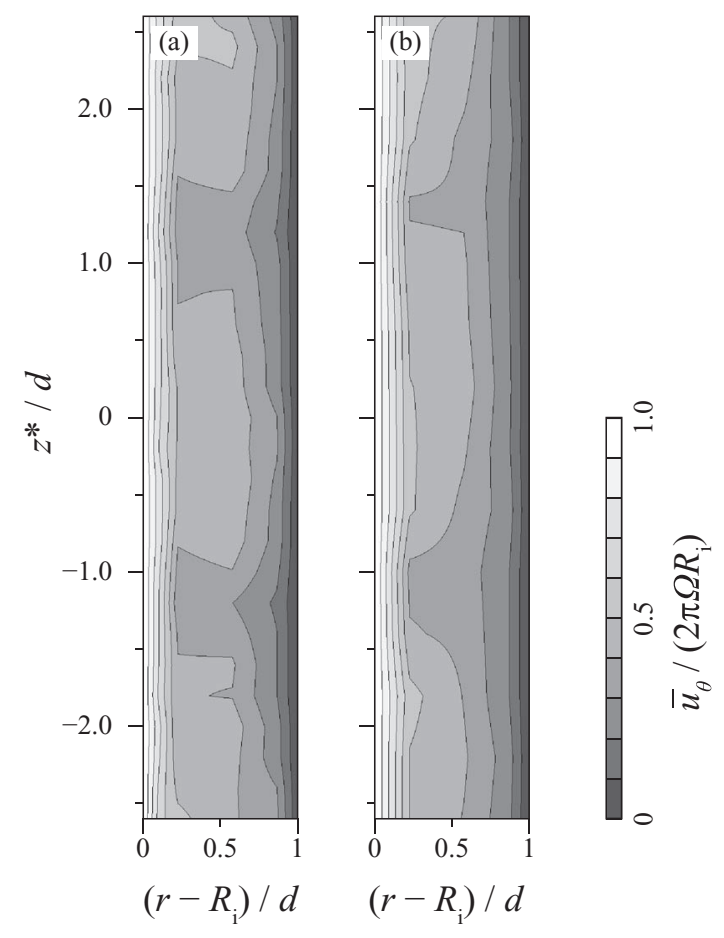

FIG. 10. Time-averaged azimuthal velocity components for (a) single phase and (b) two-phase flow $\left(\alpha=1.2 \times 10^{-4}\right)$ at $R e^{*}=8$. 

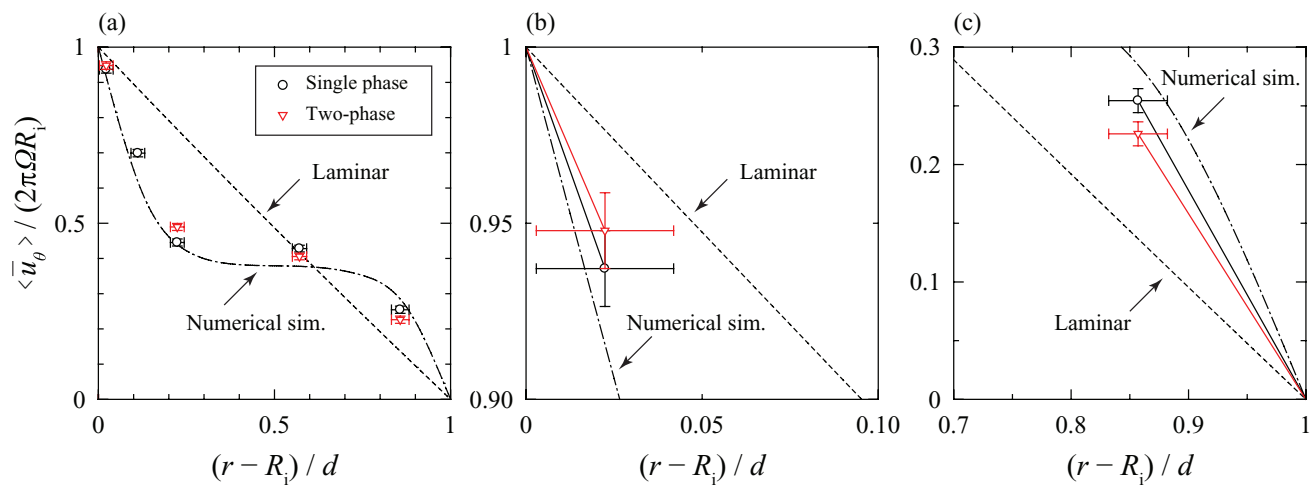

FIG. 11. Comparison of time-averaged azimuthal velocity data at $R e^{*}=8$ : (a) whole of the fluid layer, near the (b) inner and (c) outer cylinder. Symbols indicate single phase flow (circles) and two-phase flow (triangles, $\alpha=1.2 \times 10^{-4}$ ), and lines indicate the velocity profiles of numerical simulation (DNS) and the laminar flow (i.e., analytical solution for circular Couette flow, CCF, short dashed line). Error bars indicate the spatial (horizontal bar) and velocity resolution (vertical bar).

region and are reduced at the lower region of the out-flow region. The azimuthal flow structure was made asymmetrically distorted about the out-flow region in the axial/gravitational direction by the addition of microbubbles (Fig. 10(b)). Figure 11(a) shows the axial and temporally averaged azimuthal velocity profiles along the radial direction. The velocity gradient decreases monotonically in the radial direction in the two-phase condition. The laminar profile (CCF, dashed line) is displayed as references. The numerical profile obtained from Direct Numerical Simulation (DNS) for a single phase flow that authors calculated by our own code (dashed-dotted line) shows the feature of the distortion in the azimuthal velocity profile. The azimuthal velocity distribution is distorted from the CCF and becomes a stepper shape, which agrees well with that in a similar experimental configuration. ${ }^{45}$ The gradient of the azimuthal velocity profile, $-\frac{\partial}{\partial r}\left\langle\overline{u_{\theta}}\right\rangle$, near the inner and outer walls can be obtained from the velocity difference between the wall velocity and measurement data, and is shown in Figs. 11(b) and 11(c). In the figures, the lines connecting the wall and symbols indicate the velocity gradient. The velocity gradient near the walls that provides the wall shear stress, $\tau_{\text {wall }}\left(=-\left.\mu \frac{\partial}{\partial r}\left\langle\overline{u_{\theta}}\right\rangle\right|_{\text {wall }}\right)$, decreases by approximately $10 \%$ near both the inner and outer cylinder walls in the two-phase condition (Table III). These decreases of the mean azimuthal velocity gradient near the walls by the addition of microbubbles are equivalent to decreasing the Reynolds number at the flow regime below the turbulent Taylor vortex flow. ${ }^{45}$

Momentum transfer in supercritical Taylor-Couette flows is conducted mainly by radial flows due to the appearance of Taylor vortices: The out-flow carries high-speed azimuthal flows from near the inner cylinder to the outer cylinder and the low-speed azimuthal flows are carried by the radial counter flows, and vice versa. The Taylor vortex tube array exhibits the axial oscillation propagating in the azimuthal direction, owing to the instabilities of the out flow jet ${ }^{37}$ or shear instabilities of the azimuthal velocity in the axial direction. ${ }^{38-40}$ The general mechanism of the appearance of the azimuthal wave is still an open problem. The roots of the waviness, however, are definitely linked to the hydrodynamic instability of the velocity gradient in the out-flow region. Wave modulations appear as the result of the nonlinear development of the waves at high Reynolds number regimes. These results suggest that the reduction in the wave modulation (see Sec. IV B) is due to the decrease in the instabilities related with the spatial velocity gradient in the out-flow regions. Figures 12(a) and 12(b) show schematic outlines of the microbubble distribution in the wavy vortex structure on the rotating frame and the momentum transfer in the fluid layer under bubbly conditions, respectively. The momentum exchange is enhanced by the preferential distribution and migration of microbubbles swarming near the out-flow region and near the inner cylinder, which supply the momentum into the fluid. The fluctuation of the azimuthal velocity of the basic power, $\left\langle\tilde{u}_{\theta}\right\rangle\left(f_{\mathrm{w}}\right)$, is also decreased (see Table III), because migration of bubble swarms shifts the direction of the high-speed azimuthal flows to the axial direction. The flow elements naturally appear owing to the hydrodynamic instabilities with the increase in the Reynolds number in Taylor-Couette flows. 
(a)

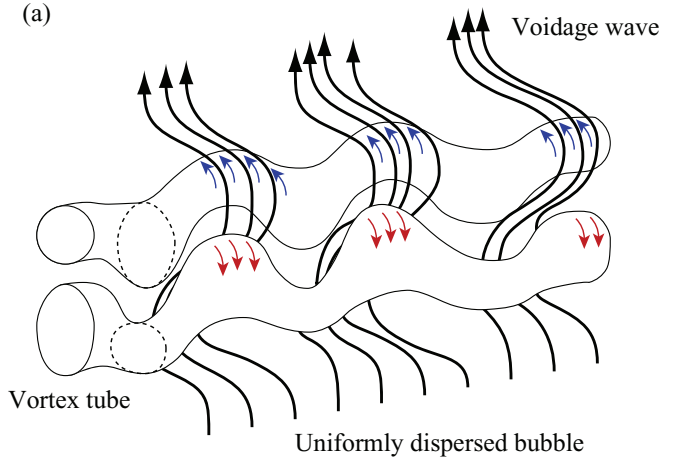

(b)

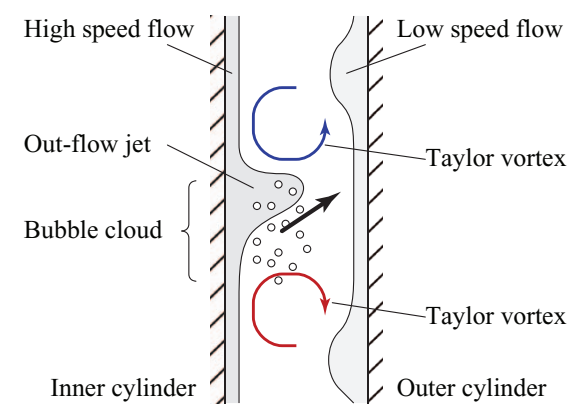

FIG. 12. Schematic outline of (a) microbubble distribution in wavy Taylor vortex tube. Red and blue arrows indicate rotational direction of vortices; black arrows indicate the motion of microbubbles. (b) Rising motion of microbubble cloud in the cross section of Taylor vortices.

The external forcing from oscillating cylinders modifies the onset of the appearance of the primal instability ${ }^{46}$ or energy concentration ${ }^{47}$ of a flow mode. The modifications of flow elements shown in Table III, i.e., intensification/attenuation of waviness and relaxation of the velocity gradient, represent energy conversions within flow modes. The spatial distribution pattern of the buoyant force due to the inhomogeneously distributed microbubbles is generated by the interaction from the spatially and temporally periodic flow pattern of the surrounding liquid phase. This non-uniform external forcing of microbubbles has modified the liquid phase flow as oscillating cylinders do. ${ }^{46,47}$

\section{CONCLUSION}

The effects of the presence of microbubbles with $O(10 \mu \mathrm{m})$ mean diameter on the coherent vortical structures and the flow transition were investigated in a vertical Taylor-Couette system in laminar-transition regions corresponding to wavy/modulated-wavy Taylor vortex flow regimes. The addition of uniformly dispersed microbubbles was not expected to modify any fluid phenomena, since the variations in the fluid characteristics, i.e., the density and viscosity of the bulk, and the external energy input by the bubbles forcing are small enough even at the case at the maximum void fraction, $\alpha=1.2 \times 10^{-4}$. However, our experimental results indicated considerable modifications of the flows, particularly at the flow transition of the system.

The first finding was that microbubbles form voidage waves in the azimuthally traveling waviness; the number density of the microbubbles increases at the crest of the waves and decreases at the trough. Further, highly concentrated microbubbles migrate in the axial streams that connect successive counter-rotating Taylor vortices. In contrast with other studies, ${ }^{21,22,26,27}$ the presence of microbubbles in our experiments does not modify the flow into new flow states as expected, because of the smaller diameter and lower void fractions. The axial wavelength of the Taylor vortices, frequency of waves, number of azimuthal waves, and traveling velocity of waves were unchanged in two-phase conditions compared to the single phase state. However, the voidage wave, whose structure conforms to the basic azimuthal wave, affected on the waviness. The power of the basic wave fluctuation is enhanced by the addition of microbubbles. On the other hand, the modulation on the basic wave is reduced. Heavy solid particles also show organized distributions of number concentrations in flows. ${ }^{48,49}$ Bosse and Kleiser ${ }^{48}$ presented the heavy particle motion simulated by a point-force coupling model with an Eulerian-Lagrangian LES in an isotropic homogeneous turbulence: The particle concentration distribution is formed by the vortical structure, and thus highly concentrated heavy particles preferentially settle down in the downward flow region of the continuous phase. Further, in settling, the preferentially dispersed particles selectively modify the flow structure, as represented in the power spectra of the spatial velocity fluctuation. The flow regime where we conducted experiments is generally not a turbulent flow but a primal time-dependent flow in the so called transition regime. The frequency components of the power spectra for the velocity fluctuation, however, are also selectively modified by the organized microbubble distribution in our experiments. 
Intensification and attenuation of wave components by the addition of microbubbles obey a simple law, i.e., the dominant wave represented by the strongest power in the spectra is enhanced, and other weaker waves are diminished. The detailed mechanisms for generating voidage waves conforming to the azimuthal wave are not fully understood yet and require further investigation.

The second finding is that microbubbles modify not only the flow structure in the buoyant direction but also that in the direction perpendicular to the buoyant direction. The spatial gradient of the tangential velocity distribution is decreased by the addition of microbubbles. Typically, the gradient in the radial direction is reduced by $10 \%$ near both the inner and outer cylinder walls in the two-phase condition. This suggests that the wall shear stress may be reduced $10 \%$ by the addition of microbubbles even at a very small void fraction, $\alpha=1.2 \times 10^{-4}$. Bubbles in the horizontal channels accumulate in the region so-called "sweep" or "ejection" region; they modify the role of the momentum exchange described as the Reynolds shear stress ${ }^{5,7,15}$ and displace the streamwise vortical structures away from the wall because of the gravity force. ${ }^{15}$ The reduction of the wall shear stress as the gradient in the streamwise velocity is thought to be resulted from the modification flow structure in the direction of gravity. ${ }^{16}$ Gravitational force did not displace the flow structures in the vertical Taylor-Couette system, unlike the case in horizontal channel flows. The secondary wavy structure, however, is vertically modified by the basic oscillation whose power is intensified in the direction of gravity. The momentum exchange in supercritical Taylor-Couette flows is conducted mainly by Taylor vortices: the secondary flow (the radial out-flow jet) carries high-speed fluids from near the inner cylinder to the outer cylinder. Microbubble migration in the axial flow is proposed to make shift the flow direction of the fluid having high azimuthal velocity to the gravitational/axial direction. The axial oscillation of the basic wave is simultaneously enhanced. Further, the modulation wave resulting from the instability due to large velocity gradients is reduced because the gradient of the azimuthal velocity is relaxed by this increase in the mixing effect with the addition of microbubbles. The modification of the secondary wavy structure reaches a global shear state, even when the direction of gravity direction is perpendicular to the direction of the main flow, "sweep," or "ejection."

To summarize, the present experiments demonstrated several modifications of flows with the addition of microbubbles; intensification of the basic azimuthal wave, attenuation of the wave modulation, and a decrease in the spatial gradient of the azimuthal velocity. These modifications induce a pseudo-decrease in the effective Reynolds number with a tiny void fraction. Similar modification of the global flow field, such as a decrease in the effective Reynolds number or a delay in the flow transitions, has also been reported. ${ }^{4,6,8,50}$ A coherent flow structure near the wall, the source of global momentum transfer, also still exists at the flow regime on which those studies focused. In shear flows containing microbubbles including open systems such as channel flows, the interaction between organized distributions of microbubbles and the coherent secondary wavy structures would results in modifications of the global flow state like those in our investigations.

\section{ACKNOWLEDGMENTS}

We acknowledge Professor Dr. Dr. Y. Takeda, Professor Emeritus of Hokkaido University, and Dr. K. Sugiyama, The University of Tokyo, for giving us essential information about this study. We also want to thank Dr. S. Yoshida for giving kind lessons on how to perform LDA measurements. This study was funded by a Grant-in-Aid for JSPS Fellows No. 24 · 184 and a JSPS Grant-in-Aid for Scientific Research, No. A 24246033. The PIV programs used in this study were developed by the LFC-PIV project, Hokkaido University.

\section{APPENDIX: DERIVATION OF THE KOLMOGOROV LENGTH SCALE}

The derivation of Eq. (6) is given below. The empirical relation of the dimensionless torque, $G$, as a function of the Reynolds number, $R e$, is given by $\mathrm{Wendt}^{51}$ as

$$
G=\frac{T}{\rho v^{2} h}=1.45 \frac{(\operatorname{Re} \eta)^{\frac{3}{2}}}{(1-\eta)^{\frac{7}{4}}},
$$


where $T, \rho, v, h$, and $\eta$ are the torque acting on the inner axis, the density and kinematic viscosity of the test fluid, height of the cylinders, and the radius ratio of the cylinders, respectively. The torque is related to the spatio-temporal averaged energy dissipation rate, $\langle\bar{\varepsilon}\rangle$ as follows:

$$
\langle\bar{\varepsilon}\rangle=\frac{T U_{\text {wall }}}{\pi \rho\left(R_{\mathrm{o}}^{2}-R_{\mathrm{i}}^{2}\right) h},
$$

where $U_{\text {wall }}, R_{\mathrm{i}}$, and $R_{\mathrm{o}}$ are the inner cylinder wall velocity, and inner and outer cylinder radii, respectively. The Kolmogorov length scale, $l$, is given as

$$
l=\left(\frac{v^{3}}{\langle\bar{\varepsilon}\rangle}\right)^{\frac{1}{4}} .
$$

Substituting (A1) and (A2) into (A3), we obtain Eq. (6) in Sec. II B.

${ }^{1}$ S. L. Cessio, "Frictional drag reduction of external flow with bubble and gas injection," Annu. Rev. Fluid Mech. 42, 183-203 (2010).

${ }^{2}$ M. E. McCormick and R. Bhattacharyya, "Drag reduction of a submersible hull by electrolysis," Nav. Eng. J. 85, 11-16 (1973).

${ }^{3}$ Y. A. Hassan, C. C. Gutierrez-Torres, and J. A. Jimenez-Bernal, "Temporal correlation modification by microbubbles injection in a channel flow,” Int. Commun. Heat Mass Transf. 32, 1009-1015 (2005).

${ }^{4}$ Y. Murai, Y. Oishi, T. Sasaki, T. Kodama, and F. Yamamoto, "Turbulent shear stress profile in horizontal bubbly channel flow," in Proceedings of the 6th International Symposium on Smart Control of Turbulence, Tokyo (2005), pp. 289-295.

${ }^{5}$ Y. Murai, Y. Oishi, Y. Takeda, and F. Yamamoto, "Turbulent shear stress profiling in a bubbly channel flow assessed by particle tracking velocimetry," Exp. Fluids 41, 343-352 (2006).

${ }^{6}$ J. Ortiz-Villafuerte and Y. A. Hassan, "Investigation of microbubble boundary layer using particle tracking velocimetry," J. Fluids Eng. 128, 507-519 (2005).

${ }^{7}$ K. Hara, T. Suzuki, and F. Yamamoto, "Image analysis applied to study on frictional-drag reduction by electrolytic microbubbles in a turbulent channel flow," Exp. Fluids 50, 715-727 (2011).

${ }^{8}$ B. Jacob, A. Olivieri, M. Miozzi, E. F. Campana, and R. Piva, "Drag reduction by microbubbles in a turbulent boundary layer," Phys. Fluids 22, 115104 (2010).

${ }^{9}$ H. H. Legner, "A simple model for gas bubble drag reduction,” Phys. Fluids 27, 2788-2790 (1984).

${ }^{10}$ G. I. Taylor, "The viscosity of a fluid containing small drops of another fluid," Proc. R. Soc. London, Ser. A 138, 41-48 (1932).

${ }^{11}$ G. K. Batchelor, An Introduction to Fluid Dynamics (Cambridge University Press, Cambridge, 1967).

${ }^{12}$ V. S. L'vov, A. Pomyalov, I. Procaccoia, and V. Tiberkevich, "Drag reduction by microbubbles in turbulent flows: The limit of minute bubbles," Phys. Rev. Lett. 94, 174502 (2005).

${ }^{13}$ K. Sugiyama, E. Calzavarini, and D. Lohse, "Microbubbly drag reduction in Taylor-Couette flow in wavy vortex regime," J. Fluid Mech. 608, 21-41 (2008).

${ }^{14}$ J. Xu, M. R. Maxey, and G. E. Karniadakis, "Numerical simulation of turbulent drag reduction using micro-bubbles," J. Fluid Mech. 468, 271-281 (2002).

${ }^{15}$ A. Ferrante and S. Elghobashi, "On the physical mechanisms of drag reduction in a spatially developing turbulent boundary layer laden with microbubbles," J. Fluid Mech. 503, 345-355 (2004).

${ }^{16}$ A. Ferrante and S. Elghobashi, "Reynolds number effect on drag reduction in a microbubble-laden spatially developing turbulent boundary layer," J. Fluid Mech. 543, 93-106 (2005).

${ }^{17}$ M. Couette, "Etude sur le frottement des liquides," Ann. Chim. Phys. 21, 433 (1890).

${ }^{18}$ G. I. Taylor, "Stability of a viscous liquid contained between two rotating cylinders," Philos. Trans. R. Soc. London, Ser. A 223, 289-343 (1923).

${ }^{19}$ C. D. Andereck, S. S. Liu, and H. L. Swinney, "Flow regimes in a circular Couette system with independently rotating cylinders," J. Fluid Mech. 164, 155-183 (1986).

${ }^{20}$ E. L. Koschmieder, Bénard Cells and Taylor Vortices (Cambridge University Press, New York, 1993).

${ }^{21}$ Y. Shiomi, H. Kutsuna, K. Akagawa, and M. Ozawa, "Two-phase flow in an annulus with a rotating inner cylinder (flow pattern in bubbly flow region)," Nucl. Eng. Des. 141, 27-34 (1993).

${ }^{22}$ H. Djeridi, C. Gabillet, and Y. Billard, "Two-phase Couette-Taylor flow: Arrangement of the dispersed phase and effect on the flow structure," Phys. Fluids 16, 128-139 (2004).

${ }^{23}$ T. H. van den Berg, S. Luther, D. Lathrop, and D. Lohse, "Bubbly turbulent drag reduction is a boundary effect," Phys. Rev. Lett. 98, 084501 (2007).

${ }^{24}$ E. Climent, M. Simonnet, and J. Magnaudet, "Preferential accumulation of bubbles in Couette-Taylor flow patterns," Phys. Fluids 19, 083301 (2007).

${ }^{25}$ T. H. van den Berg, S. Luther, D. P. Lathrop, and D. Lohse, "Drag reduction in bubbly Taylor-Couette turbulence," Phys. Rev. Lett. 94, 044501 (2005).

${ }^{26}$ Y. Murai, H. Oiwa, and Y. Takeda, "Frictional drag reduction in bubbly Taylor-Couette flow," Phys. Fluids 20, 034101 (2008).

${ }^{27}$ K. Yoshida, Y. Tasaka, Y. Murai, and Y. Takeda, "Mode transition in bubbly Taylor-Couette flow measured by PTV," J. Phys.: Conf. Ser. 147, 012013 (2009). 
${ }^{28}$ J. Lu, A. Fernández, and G. Tryggvason, “The effect of bubbles on the wall drag in a turbulent channel flow," Phys. Fluids 17, 095102 (2005).

${ }^{29}$ T. S. Lo, V. S. L’vov, and I. Procaccia, "Drag reduction by compressible bubbles,” Phys. Rev. E 73, 036308 (2006).

${ }^{30}$ R. C. Di Prima and H. L. Swinney, "Instabilities and transition in flow between concentric rotating cylinders," Top. Appl. Phys. 45, 139-180 (1985).

${ }^{31}$ Y. Takeda, "Quasi-periodic state and transition to turbulence in a rotating Couette system," J. Fluid Mech. 389, 81-99 (1999).

${ }^{32}$ T. Watamura, Y. Tasaka, and Y. Murai, "Quantitative visualization of microbubble streams in Taylor vortices," Theor. Appl. Mech. Jpn. 61, 123-131 (2013).

${ }^{33}$ T. Ido, Y. Murai, and F. Yamamoto, "Postprocessing algorithm for particle-tracking velocimetry based on ellipsoidal equations," Exp. Fluids 32, 326-336 (2002).

${ }^{34}$ Y. Takeda, "Velocity profile measurement by ultrasound Doppler shift method," Int. J. Heat Fluid Flow 7, 313-318 (1986).

${ }^{35}$ Y. Takeda, W. E. Fischer, K. Kobashi, and T. Takada, "Spatial characteristics of dynamic properties of modulated wavy vortex flow in a rotating Couette system," Exp. Fluids 13, 199-207 (1992).

${ }^{36}$ M. Gorman and H. L. Swinney, "Spatial and temporal characteristics of modulated waves in the circular Couette system," J. Fluid Mech. 117, 123-142 (1982).

${ }^{37}$ M. Marcus, "Simulation of Taylor-Couette flow. Part 2. Numerical results for wavy-vortex flow with one travelling wave," J. Fluid Mech. 146, 65-113 (1984).

${ }^{38}$ C. A. Jones, "Transition to wavy Taylor vortices,” J. Fluid Mech. 157, 135-162 (1985).

${ }^{39}$ K. T. Coughlin and P. S. Marcus, "Modulated waves in Taylor-Couette flow Part 2. Numerical simulation," J. Fluid Mech. 234, 19-46 (1992).

${ }^{40}$ A. Akonur and R. M. Lueptow, “Three-dimensional velocity field for wavy Taylor-Couette flow," Phys. Fluids 15, 947-960 (2003).

${ }^{41}$ L. D. Landau and E. M. Lifshitz, Fluid Mechanics - Course of The Theoretical Physics (Pergamon Press, Bristol, 1959), pp. 102-107.

${ }^{42}$ G. P. Neitzel, "Numerical comparison of time-dependent Taylor-vortex flows in finite-length geometries," J. Fluid Mech. 141, 51-66 (1984).

${ }^{43}$ S. T. Wereley and R. M. Lueptow, "Spatio-temporal character of non-wavy and wavy Taylor-Couette flow," J. Fluid Mech. 364, 59-80 (1998).

${ }^{44}$ P. Ashwin and G. P. King, "A study of paths in non-axisymmetric Taylor-Couette flows," J. Fluid Mech. 338, 341-362 (1997).

${ }^{45}$ S. T. Wereley and R. M. Lueptow, “Azimuthal velocity in supercritical circular Couette flow," Exp. Fluids 18, 1-9 (1994).

${ }^{46}$ T. J. Walsh, W. T. Wagner, and R. J. Donnelly, "Stability of modulated Couette flow," Phys. Rev. Lett. 58, 2543-2546 (1987).

${ }^{47}$ Y. Takeda, M. Morinaga, and Y. Tasaka, “Oscillating Taylor-Couette flow (Azimuthal motion)," J. Phys.: Conf. Ser. 137, 012002 (2008).

${ }^{48}$ T. Bosse and L. Kleiser, "Small particles in homogeneous turbulence: Settling velocity enhancement by two-way coupling," Phys. Fluids 18, 027102 (2006).

${ }^{49}$ K. Yeo, S. Dong, E. Climent, and M. R. Maxey, "Modulation of homogenous turbulence with finite size bubbles or particles," Int. J. Multiphase Flow 36, 221-233 (2010).

${ }^{50}$ A. Serizawa, T. Inui, and T. Eguchi, "Flow characteristics and pseudo-laminarization of vertically up-ward air-water milky bubbly flow with micro bubble in a pipe," J. Jpn. Soc. Multiphase Flow 19, 335-343 (2005) [Konsoryu 19, 335-343 (2005) (in Japanese)].

${ }^{51}$ F. Wendt, “Turbulente Strömungen zwischen zwei rotierenden konaxialen,” Zylindern. Ing.-Arch. 4, 577-595 (1933). 